\title{
Vivid Visualization in the Experience of Phobia in Virtual Environments: Preliminary Results
}

\author{
MILTON P. HUANG, M.D., JOSEPH HIMLE, Ph.D., and NORMAN E. ALESSI, M.D.
}

\begin{abstract}
We explored the effect of being able to form vivid mental images on the experience of phobia during exposure treatment in virtual environments. Taking subjects with acrophobia, we randomized them to two treatment groups: in vivo exposure treatment in a real building versus virtual exposure in a model of the same building, projected in a CAVETM Virtual Environment. Using Marks' Vividness of Visual Imagery Questionnaire (VVIQ-2) as a measure of vividness of visual imagery, we performed Pearson correlations of vividness with amount of fear experienced as measured by Pekala's Phenomenology of Consciousness Inventory (PCI). Contrary to expectation, we found a negative correlation between vividness of visualization and amount of fear experienced during exposure $(R=-0.77728, p=0.0137)$. There was a positive correlation between fear and vividness of visualization during the exposure experience as measured by the PCI $(R=0.94083, p=0.0171)$. These results are discussed in terms of possible differences between the VVIQ and PCI vividness measures as well as possible effects from the subject's experience.
\end{abstract}

\section{INTRODUCTION}

$\mathbf{V}$ IRTUAL ENVIRONMENTS (VEs) are being broadly applied in the clinical treatment of psychiatric disorders. The successful application of a VE for such purposes will depend on its ability to create a specific emotional response. ${ }^{1}$ Although clinical trials and case reports support the argument that VEs are effective in the treatment of phobias, ${ }^{2,3}$ few have explored the question of what psychological factors determine who will respond emotionally to a virtual phobic experience. Most of the studies that attempt to determine the impact of VEs have measured the "sense of presence" one has in the VE, with the implicit assumption that the more one feels that they are "really there" in the environment, the more they will respond as they would in re- ality. ${ }^{4,5}$ We sought to determine if other factors such as the vividness of visualization might influence the emotional experience of virtual reality, expecting that a tendency to visualize more vividly would correlate to a more intense experience. We used as our model the emotional experience of exposure treatment for acrophobia, examining fear response to treatment in a real building and in the same building replicated in a virtual environment. ${ }^{6}$

\section{MATERIALS AND METHODS}

\section{Subjects}

Subjects were recruited through advertisements for volunteers with a fear of looking out 
of windows of tall buildings. They were screened by the Structured Clinical Interview for DSM-IV Axis I Disorders-Patient Edition $(\mathrm{SCIC}-\mathrm{IV})^{7}$ to ensure they met criteria for the specific phobia. Subjects with psychotic symptoms were excluded from the study. All subjects also completed an initial questionnaire package including the Beck Depression Inventory (BDI), ${ }^{8}$ Marks' Vividness of Visual Imagery Questionnaire (VVIQ-2), ${ }^{9}$ and Tellegen's Multidimensional Personality Questionnaire (MPQ). ${ }^{10}$

\section{Measures}

The VVIQ is a second generation instrument that has been in use for more than 25 years. It quantifies the vividness of a subject's visual imagery by having them rate the vividness of eight mental images they form as they complete the questionnaire. Summarizing and reanalyzing the results of several dozen studies on the VVIQ, McKelvie ${ }^{11}$ produced the following figures for its reliability: internal consistency $=0.88$, test-retest $=0.74$, immediate $=$ 0.64 , delayed alternate form $=0.54$. The lower values for the last three are likely due in part to the dynamic nature of "vividness." This test has also been shown to be subject to social desirability effects in at least four studies, although some studies suggest that these results are not due to impression management demands but, rather, to other factors such as welladjustedness. ${ }^{12}$ In order to assess such potential factors, we also included the BDI and the MPQ.

The BDI is a 21-question inventory of depressive symptoms that has high correlations with clinical ratings of depression. ${ }^{8}$ It has been examined extensively and has been found to have excellent internal consistency and concurrent validity. ${ }^{13,14}$

The MPQ is a factor-analytically developed self-report instrument that assesses self-view of personality along eleven primary dimensions and three higher order traits. Constructs were reiteratively reassessed and refined through factor analyses. The eleven primary scales are: (1) wellbeing, (2) social potency, (3) achievement, (4) social closeness, (5) stress reaction, (6) alienation, (7) aggression, (8) control vs. im- pulsiveness, (9) harm avoidance, (10) traditionalism, and (11) absorption. The higher order traits are positive affectivity, negative affectivity, and constraint. Along with these measures, the MPQ produces six validity scales including associative slips, unlikely virtues, desirable response inconsistency, true response inconsistency, variable response inconsistency, and an overall index of invalid response. In our analysis, we excluded all data for subjects with an overall invalid index over 37.

Our primary dependent measure was the Phenomenology of Consciousness Inventory (PCI). ${ }^{15}$ This is a third generation self-report questionnaire that assesses twelve major dimensions and fourteen subdimensions of conscious experience. Dimensions were developed from theory, exploratory factor analyses, and confirmatory cluster analyses. Items were refined through reiterative reliability correlation analyses. The resulting dimensions (and associated subdimensions) are (1) altered experience (body image, time sense, unusual meanings, perception); (2) positive affect (joy, sexual excitement, love); (3) negative affect (fear, anger, sadness); (4) attention (direction, absorption); (5) imagery (amount, vividness); (6) self-awareness; (7) state of awareness; (8) internal dialogue; (9) rationality; (10) volitional control; (11) memory; and (12) arousal. The questionnaire contains 53 questions that present dipolar statements that are marked along a 0-6 scale. It also provides a reliability score of the subjects' responses. In this study, all measures with a reliability score greater than 2 were excluded from analysis.

\section{Procedure}

All subjects were randomized to treatment in reality or virtual reality. Exposure treatment for both groups occurred in a single 90-minute session following the same exposure protocol. All subjects wore similar virtual reality LCD shutter glasses. Therapist-assisted exposure sessions (real or virtual) began with the subject and the therapist looking from a first floor window while the therapist encouraged the subject to look at the height outside. Once the subject reported low levels of anxiety at floor one, the treatment progressed to higher and higher 
floors according to a defined exposure protocol based on the subject's subjective rating of fear on a 1-10 scale. The exposure protocol (in vivo or virtual) was 90 minutes in duration. If the subject reached the top of the building (the eighth floor) before 90 minutes had elapsed, he or she would be asked to continue looking out of the eighth floor window until the session was complete. After exposure, the subject would then complete a questionnaire package on their experience, including measures of physical discomfort ${ }^{16}$ and the Phenomenology of Consciousness Inventory (PCI).

The VE was experienced in a CAVE (CAVE Automatic Virtual Environment) ${ }^{17}$ with three $10 \times 10$-foot walls and a $10 \times 10$-foot floor for projection surfaces. The four $1024 \times 768$ resolution images were generated by a Silicon Graphics Onyx 2 computer. The virtual model was designed to simulate the same view from the real building, by modeling and creating texture maps for the elevator, elevator lobby, and for the buildings and trees in the courtyard visible from the exposure window. The simulation also included a virtual elevator to replicate the entire in vivo experience as closely as possible.

\section{RESULTS}

Mean values of dependent measures of interest are given in Table 1, divided according to virtual or in vivo exposure. The mean fear score was significantly higher in the in vivo group than the virtual group ( $p=0.0418$ for $\mathrm{t}$ test, and $p=0.0317$ for one-sided Wilcoxon). Altered experience was significantly different between treatment groups as well $(p=0.0181$ for t-test and $p=0.0317$ for one-sided Wilcoxon). We analyzed significance of these variables by determining the Pearson correlation coefficients between these dependent mea- sures and the visualization score from the VVIQ (Table 2).

Despite high coefficients, correlation was not significant for either the virtual or the in vivo groups because of the small sample size. Pooling the results from both in vivo and virtual subjects produced a significant correlation between experienced fear and vividness of visualization $(R=0.77728, p=0.0137)$. This is a negative correlation, graphically depicted in Figure 1 using a linear regression and scatterplot. The PCI vividness of imagery scores correlated well with fear scores $(R=0.94083, p=$ $0.0171)$ and also had a negative correlation with vividness from the VVIQ $(R=-0.56581, p=$ 0.1123).

"Arousal" on the PCI measures muscular tension, using dipoles such as, "I felt no feelings of tension or tightness at all" vs. "I felt tense and tight." It correlated with experienced fear over the pooled sample $(R=0.71655, p=$ 0.0299 ), and also correlated negatively with the VVIQ results in the pooled sample $(\mathrm{R}=$ $-0.77840, p=0.0135$ ) (also shown in Table 2).

\section{DISCUSSION}

Because of the low number of subjects (4-5 in each treatment group and 9 in the pooled group), these results must be seen as preliminary. Our correlations may become insignificant if we find there has been significant bias to our sample. Similarly, a higher number of subjects may make our correlations significant in each of our treatment groups.

We expected that vividness of visualization would increase fear and arousal scores, and, indeed, the measurement of vividness during the experience using the PCI did correlate. Why this would correlate negatively with our scores from the VVIQ is uncertain. From the way the

Table 1. Mean Values of Dependent Variables

\begin{tabular}{|c|c|c|c|c|c|c|c|c|}
\hline & \multicolumn{2}{|c|}{ Fear from $P C I$} & \multicolumn{2}{|c|}{ Arousal from PCI } & \multicolumn{2}{|c|}{ Altered experience from PCI } & \multicolumn{2}{|c|}{ Attention from PCI } \\
\hline & Virtual & In vivo & Virtual & In vivo & Virtual & In vivo & Virtual & In vivo \\
\hline deviation) & $\mathrm{SD}=1.68$ & $\mathrm{SD}=0.816$ & $\mathrm{SD}=2.51$ & $\mathrm{SD}=0.750$ & $\mathrm{SD}=0.172$ & $\mathrm{SD}=0.192$ & $\mathrm{SD}=0.43$ & $\mathrm{SD}=0.117$ \\
\hline
\end{tabular}




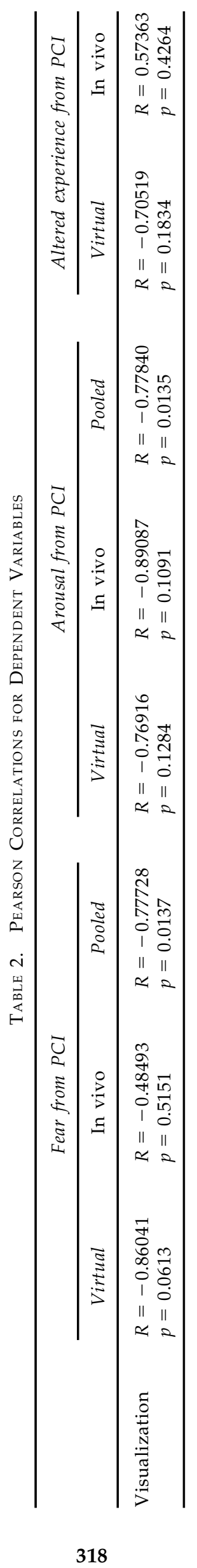




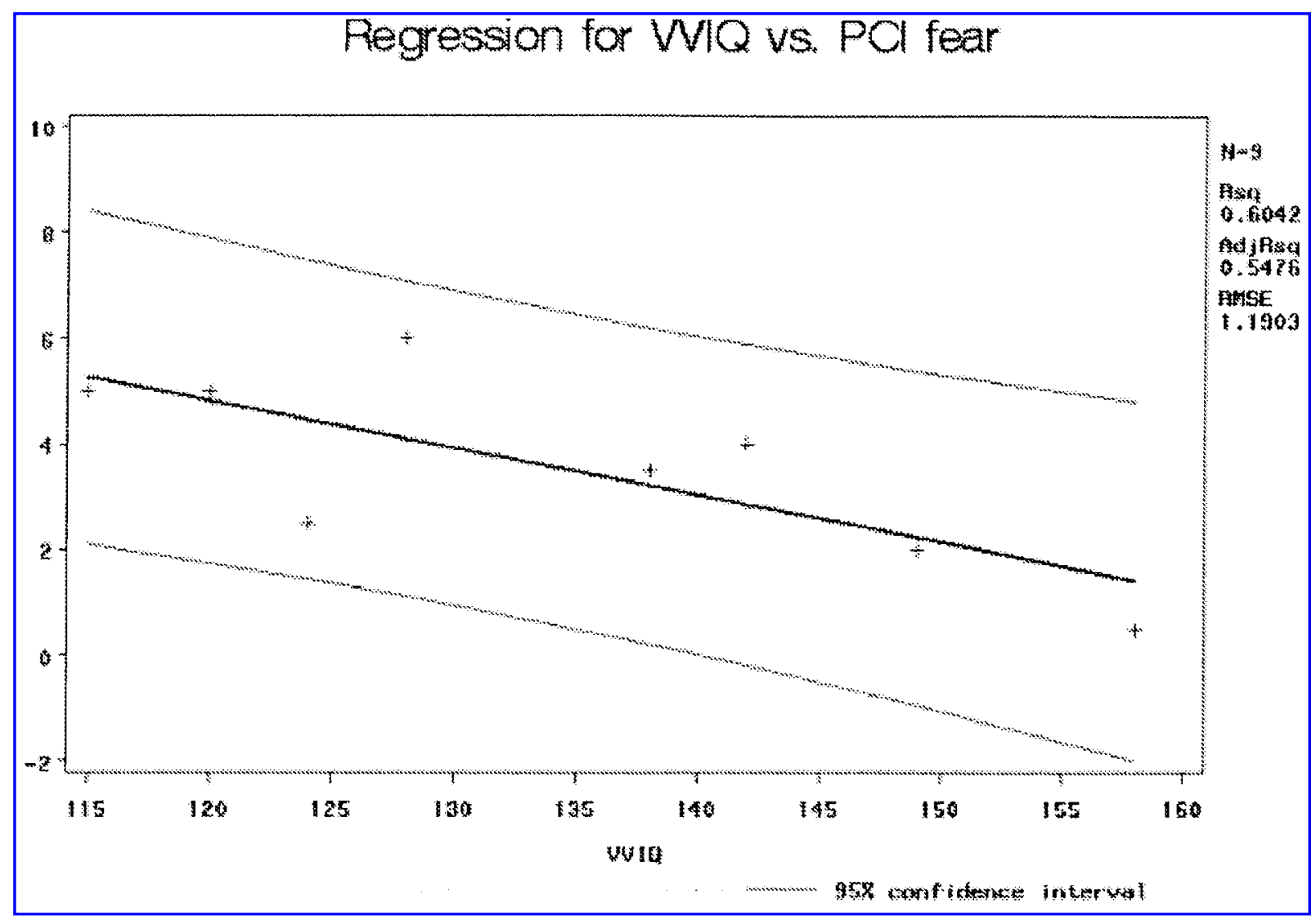

FIG. 1. Linear regression of vividness score versus fear score.

questionnaire is structured, it is possible that the VVIQ measures a person's ability to mentally form vivid and clear images in their imagination, which differs from the reported experience of vivid visualization during exposure when subjects are actively told to look out the window and focus on the feared height. In such a case, fear would increase the active experience of vivid visualization (as measured by the PCI), but a person's ability to vividly imagine (as measured by the VVIQ) might in some way help them decrease their experience of fear by serving as a distraction. This hypothesis may also be supported by the fact that the correlation was stronger in the virtual experience $(R=$ $-0.860)$ instead of the real one $(R=-0.485)$, as one might expect that distraction by visual imagination would play a greater role in a virtual situation. Clearly, further research is needed to examine the validity of this possibility by determining what subjects are visual- izing during exposure and how that content affects their experience of fear.

A related possibility is that the VVIQ is measuring a potential to lose oneself in imagination. Some studies have suggested that a vivid and absorbed imagination style correlates with vividness as measured by the VVIQ as well as factors such as hypnotic susceptibility. ${ }^{18}$ Indeed, the MPQ's absorption scale (also referred to as the Tellegen Absorption Scale-TAS ${ }^{19,20}$ did correlate negatively with fear although the effect was insignificant in our small virtual sample $(R=-0.268, p=0.663)$. Unfortunately, this hypothesis is contradicted to some degree by the tendency we found for "altered experience" as measured in the PCI to correlate negatively with VVIQ $(R=-0.705, p=0.183)$. This calls for further studies, perhaps exploring aspects of dissociation as well as the complex interactions of these types of factors.

A final alternative to consider is that the 
VVIQ is not measuring vividness as much as it is measuring the sense of control over visualization. ${ }^{21}$ In this case, the negative correlation simply implies that a greater control over visual imagery reduces the experience of fear. This might be supported by further examinations with instruments that could more clearly identify control over imagery.

This research presents preliminary results that indicate vividness of visualization does affect fear experienced during exposure treatment. We hope to obtain further data to present more robust results, as well as spur further exploration as to how these factors and related factors of imagination, dissociation, and absorption impact emotional experiences in reality and virtual reality.

\section{ACKNOWLEDGMENTS}

We would like to acknowledge the University of Michigan Psychiatry Research Committee, which provided grant support for this research. We would also like to acknowledge Barabara O'Keefe for use of the CAVE facility. We thank Aileen Prout, Diane Koram, and Hedieh Briggs for their work in performing SCIDS and as therapists.

\section{REFERENCES}

1. Huang, M.P., \& Alessi, N.E. (1999). Mental health implications for presence. CyberPscyhology and Behavior, 2:15-18.

2. Rothbaum, B.O., Hodges, L.F., Kooper, R., Opdyke, D., Williford, J.S., \& North, M. (1995). Effectiveness of computer-generated (virtual reality) graded exposure in the treatment of acrophobia. Am Psychiatry, 152:626-628.

3. Glantz, K., Durlach, N.I., Barnett, R.C., \& Aviles, W.A. (1997). Virtual reality (VR) and psychotherapy: Opportunities and challenges. Presence-Teleoperators and Virtual Environments, 6:87-105.

4. Huang, M.P., \& Alessi, N.E. (1999). Presence as an emotional experience. Amsterdam: IOS Press.

5. Draper, J.V., Kaber, D.B., \& Usher, J.M. (1998). Telepresence. Hum Factors, 40:354-375.

6. Huang, M.P., Himle, J., Beier, K.-P., \& Alessi, N.E. (1998). Comparing virtual and real worlds for acrophobia treatment. Amsterdam: IOS Press.

7. First, M.B., Spitzer, R.L., Gibbon, M., \& Williams, J.B. (1997). Structured clinical interview for DSM-IV Axis I
Disorders-Patient edition (SCID-I/P, version 2.0). New York: Biometrics Research, New York State Psychiatric Institute.

8. Beck, A.T. (1961). A systematic investigation of depression. Comprehensive Psychiatry, 2:163-170.

9. Marks, D.F. (1995). New directions for mental imagery research. Journal of Mental Imagery, 19:153-167.

10. Tellegen, A. (1982). Brief manual for the multidimensional personality questionnaire. Minneapolis: University of Minnesota.

11. McKelvie, S.J. (1995). The VVIQ as a psychometric test of individual differences in visual imagery vividness: A critical quantitative review and plea for direction. Journal of Mental Imagery, 19:1-106.

12. Rinaldo, R., \& Okada, R. (1993). A test of the relationship between imagery vividness and social desirability across subject selection procedures. Journal of Mental Imagery, 17:171-180.

13. Beck, A.T., Steer, R.A., \& Garbin, M.G. (1988). Psychometric properties of the Beck Depression Inventory: Twenty-five years of evaluation. Clinical Psychology Review, 8:77-100.

14. Richter, P., Werner, J., Heerlein, A., Kraus, A., \& Sauer, H. (1998). On the validity of the Beck Depression Inventory: A review. Psychopatholog y, 31;160-168.

15. Pekala, R.J. (1991). Quantifying consciousness: An empirical approach. New York: Plenum Press.

16. Kennedy, R.S., Lane, N.E., Berbaum, K.S., \& Lilienthal, M.G. (1993). Simulator Sickness Questionnaire: An enhanced method for quantifying simulator sickness. International Journal of Aviation Psychology, 3:203-220.

17. Huang, M., Couling, M., \& Alessi, N. (1998). The CAVETM System. CyberPsychology and Behavior, 1:397-399.

18. Crawford, H.J. (1982). Hypnotizability, daydreaming styles, imagery vividness, and absorption: A multidimensional study. Lournal of Personality \& Social Psychology, 42:915-926.

19. O'Grady, K.E. (1980). The absorption scale: A factoranalytic assessment. International Journal of Clinical $\mathcal{E}$ Experimental Hypnosis, 28:281-288.

20. Nadon, R., Hoyt, I.P., Register, P.A., \& Kihlstrom, J.F. (1991). Absorption and hypnotizability: Context effects reexamined. Lournal of Personality \& Social Psychology, 60:144-153.

21. Kihlstrom, J.F., Glisky, M.L., Peterson, M.A., Harvey, E.M., et al. (1991). Vividness and control of mental imagery: A psychometric analysis. Journal of Mental Imagery, 15:133-142.

Address reprint requests to: Milton Huang, M.D. 1500 E. Medical Center Dr., TC 6222/Box 0390 Ann Arbor, MI 48109-0390

E-mail: mhuang@umich.edu 
This article has been cited by:

1. Rosa Maria E.M. Costa, Luis Alfredo V. de Carvalho, Ricardo Drummond, Ana Paula T. Wauke , Marcele de Sá Guimarães . 2002. The UFRJ-UERJ Group: Interdisciplinary Virtual Reality Experiments in NeuropsychiatryThe UFRJ-UERJ Group: Interdisciplinary Virtual Reality Experiments in Neuropsychiatry. CyberPsychology Behavior 5:5, 423-431. [Abstract] [PDF] [PDF Plus] 